\title{
Perceived Effectiveness of Dairy Service Delivery Systems in Namakkal District of Tamil Nadu, India
}

\author{
S. Karthikeyan ${ }^{1 *}$, M.C. Arunmozhi Devi ${ }^{2}$, N. Narmatha ${ }^{1}$ and V. Uma ${ }^{1}$ \\ ${ }^{1}$ Department of Veterinary and Animal Husbandry Extension Education, Veterinary College \\ and Research Institute, Namakkal, Tamil Nadu, India \\ ${ }^{2}$ Dairy Extension Division, National Dairy Research Institute, Southern Regional Station, \\ Bangalore, Karnataka, India \\ *Corresponding author
}

A B S T R A C T

The present study was conducted in Namakkal District of Tamil Nadu to analyse the perceived effectiveness of various dairy service delivery systems like dairy co-operative, private integrators, public departments, private veterinarians, para-veterinarians, etc. and their extent of share in delivering the dairy services to farmers. A total of 120 respondents

\section{Keywords}

Dairy, Effectiveness, Service, Private, Cooperative, Health, Insemination

Article Info

Accepted: 04 April 2018 Available Online: 10 May 2018 were selected for the study by proportionate random sampling method and the data were collected by personal interview method using pre-tested interview schedule. Most of the respondents predominantly availed the curative ( 99.17 per cent) and preventive (95.00 per cent) services from the private veterinarians; and medicines from the pharmacies (98.33 per cent) than other dairy service delivery systems. The para-veterinarians are the major source for 75.83 per cent of the respondents for availing artificial insemination services. The respondents predominantly availed the services like concentrate feed (73.33 per cent); mineral mixture (73.33 per cent); insurance (57.78 per cent) and training and advisory services (60.00 per cent) from dairy co-operative, whereas fodder seeds and slips and credit facilities were mainly availed from neighbours (59.17 per cent) and banks (12.50 per cent) respectively. Overall it was perceived that the public department was effective in delivering heath and artificial insemination services, providing insurance and mineral mixture to the dairy farmers while the dairy co-operative was perceived to be effective in supplying fodder seeds and slips, credit facilities and training and advisory services. The commercial feed agencies were perceived as the effective service provider in supplying commercial concentrate feed than others.

\section{Introduction}

India is the largest milk producing nation in the world. The growth rate of milk production in India during the year 2015-16 was 6.28 percent with a total milk production of 155.50 million metric tonnes. Tamil Nadu produced about 7.24 million tonnes in 2015-16 contributing $4.65 \%$ to the total production of India standing in $10^{\text {th }}$ position. Among the cooperatives in India, Tamil Nadu holds $4^{\text {th }}$ place with a daily procurement of 28.50 lakh litres per day. Dairying has emerged as an important secondary source of income for millions of 
rural agricultural families and has assumed the most important role in providing employment and income generating opportunities, especially for landless, marginal and small farmers.

Dairying encompasses a chain of activities viz., milk production, processing of milk and finally marketing. For carrying out these essential activities, it requires various inputs and services which have to be delivered by external agencies. Dairy service delivery system is an agency or institution that delivers various inputs and services to the intended clientele either at free of cost or charging according to the service rendered by them. According to Umali et al., (1992), livestock services can be grouped into two major functional categories: health and production services.

Health services consist of curative services, preventive services and provision of medicines; while production services include research and extension services, provision of input such as seed, feed and artificial insemination.

There are various agencies which include public department like State Animal Husbandry Departments (SDAH), private integrators, dairy co-operative, private veterinary professionals, para-veterinary professionals, private milk vendors, educational institutes, etc. These agencies provide various services such as curative; preventive; distribution of medicines; supply of fodder seeds, slips, concentrate feed, mineral mixture; AI services; training and advisory services; insurance; and credit facilities.

Effective and efficient delivery of services by various agencies is considered as vital for sustainable dairy development. Livestock services in developed countries are in general adequately and efficiently supplied. In India, the government maintains a large public infrastructure on livestock services, but there are limitations about the service availability and utility to the livestock producers. The extent of share in delivering the services to dairy farmers has to be explored for the betterment of delivery system. Hence there is a need to understand the existing services of the agencies and their efficiency in delivering the services to dairy farmers.

\section{Materials and Methods}

The study was undertaken in Namakkal district of Tamil Nadu which falls under the Salem District Co-operative Milk Producers Union, which is the largest milk procuring union of Tamil Nadu Cooperative Milk Producers Federation. Apart from cooperative, various private diaries, vendors and cream separation units are also procuring milk from the farmers. All the four taluks of Namakkal district were purposively included for the study. For each taluk, the revenue villages which are having a female bovine population of above 1000 animals were listed out. From this list, one village having highest bovine population from each taluk was selected for the research study. Respondents were selected based on the criteria that the farmers should have milch animal either a cow or a buffalo in milking condition and availing services from different dairy service delivery systems.

The different milk procurement channels available in the selected villages were listed out. The number of dairy farmers in each milk procurement channel was listed out. By using proportionate random sampling method 30 respondents from each village were selected. Thus, a total of 120 respondents were selected for the study. The data were collected by personal interview method using pre-tested interview schedule. 


\section{Results and Discussion}

\section{Services availed from dairy service delivery systems}

The services availed by the dairy farmers from different dairy service delivery systems are presented in table 1. Most of the dairy farmers availed curative and preventive services from private veterinarians $(99.17 \%$ and $95 \%)$ followed by private integrators $(81.82 \%$ each) and dairy co-operatives $(75.55 \%$ and $84.44 \%)$. The dairy farmers were unable to take the animals to hospital during illness and the public department veterinarians were not available after their scheduled duty hours which might be the reasons for availing the services of private veterinarians. Even though the charges of private veterinarians are expensive, the farmers seek them because they reach the farmers door step was also stated by Pallavi et al., (2011) and Bardhan et al., (2015).

Majority of the dairy farmers procured medicines from pharmacies (98.33\%) followed by private veterinarians $(97.50 \%)$, private integrators $(81.82 \%)$ and dairy cooperatives $(80.00 \%)$. Researcher observed that most of the dairy farmers initially purchased medicines from pharmacies over the counter to treat the animals. After that when the ailment continues they approached either private veterinarians or other service agencies for treatment.

More than half $(59.17 \%)$ of the dairy farmers procured fodder seeds and slips from neighbours. Timely availability of quality fodder slips in sufficient quantity, easy access and visualizing the proven result in neighbours plot might be the reason for procuring from neighbours. Dairy co-operative $(33.33 \%)$ is the next available source to get fodder seeds and slips. Majority of the dairy farmers purchased concentrate feed from dairy co- operative $(73.33 \%)$, commercial feed agencies $(63.33 \%)$ and private integrators $(45.45 \%)$. Sharma (2001) also found that majority of the dairy farmers purchased concentrate feed from dairy co-operative.

Nearly three-fourth $(73.33 \%)$ of the dairy farmers purchased mineral mixture from dairy co-operative and $57.5 \%$ of them purchased directly from pharmacies. Credit facilities were availed only by meager percentage of the dairy farmers. Among those availed, $12.50 \%$ and $11.11 \%$ were availed from commercial/co-operative banks and dairy cooperative respectively. Insurance facilities were availed by $57.78 \%$ and $21.67 \%$ of the dairy farmers from dairy co-operative and public department respectively, since they were the only competent agencies for extending insurance facilities among different systems. The premium charges of insurance were deducted from the payment of milk which might have motivated the farmers to avail the service from dairy co-operative society.

AI services were predominantly availed from para-veterinarians $(75.83 \%)$ followed by private integrators $(68.18 \%)$, dairy cooperative $(57.78 \%)$ and private veterinarians $(57.50 \%)$. The para-veterinarians are readily available to the clients at their door steps with nominal charges and most of them are local persons with high credibility among the farmers.

Dairy farmers are visiting the dairy cooperative daily for milk pouring and hence most of the dairy farmers $(60 \%)$ are getting the advisory services from dairy co-operative. Rathod et al., (2012) also reported that majority of the farmers availed advisory services from dairy co-operative. Dairy farmers are also approaching public department for availing treatment and scheme facilities, might be the reasons for $28.33 \%$ 
acquiring training and advisory services from public department.

\section{Effectiveness of different dairy service delivery systems as perceived by the dairy farmers}

In a pluralistic environment where there are different dairy service providers, it is important to assess the effectiveness of the different possible providers on various criteria in order to identify the best among all. Accordingly, services delivered by more than one provider were evaluated by the dairy farmers for their perceived effectiveness. The indicators were measured on three point continuum viz. good, average and poor and the score 3, 2 and 1 was assigned respectively. The weighted percentage score was calculated for different indicators.

\section{Health services}

The perceived effectiveness of health services of dairy service delivery systems was studied and presented in table 2 . It was perceived by the dairy farmers that the curative services, preventive services and supply of medicines by private veterinarians were effective in terms of regularity (299.16, 299.12 and 299.15) and quality (299.16, 299.12 and 299.15). This might be the reason for availing the services of private veterinarians by the dairy farmers in the study area, which is in accordance with the findings of Kathiravan et al., (2011). Further, regular and quality services would enable the private veterinarians to retain their clients.

Dairy farmers perceived that public department provided cost effective curative services, preventive services and medicines (300 each) because most of the services were provided at free of cost. Further, they perceived that the timely curative (296.43) and preventive (273.75) services were provided by public department, whereas pharmacies delivered medicines (298.31) in time as they operate within the vicinity of the dairy farmers.

\section{Fodder seeds and slips}

From table 3 , it can be noticed that dairy cooperative was perceived as effective in providing fodder seeds and slips regularly (226.67) and in time (220), whereas the educational institutes (290.91 each) were effective in providing quality and variety of fodder seeds and slips. The dairy farmers also perceived that the sufficient quantity (288.73) of fodder seeds and slips can be procured from the neighbours cost effectively (300.00). Dairy co-operative maintain fodder and seed banks on contract basis so that they can provide in time regularly. The educational institutes need all varieties for academic and extension purpose and hence they maintain quality and variety of seeds and slips. In most instances, the neighbours tend to give the fodder slips to their fellow farmers at free of cost in sufficient quantity might be the reason for cost effectiveness.

\section{Concentrate feed}

From table 4, it can be inferred that the dairy farmers perceived that commercial feed agencies were effective in terms of their regularity (297.37), timeliness (297.37), quality (298.68), quantity (298.68) and type of feed (298.68). The private integrators were perceived to be cost effective (222.22). The commercial feed agencies operate in complete commercial mode and they have dealers and retailers hence they supply the required quantity of feed regularly in time based upon the need of the farmer however, the cost effectiveness was low. But the private integrators were cost effective since they are supplying the concentrate feed in non-profit motive to maintain the clients. 
Table.1 Services availed from different dairy service delivery systems

\begin{tabular}{|c|c|c|c|c|c|c|c|c|c|}
\hline $\begin{array}{c}\text { S. } \\
\text { No. }\end{array}$ & Type of services & 8̊] & 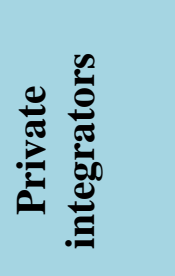 & 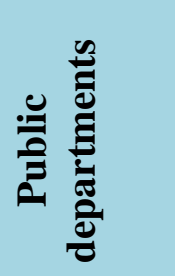 & 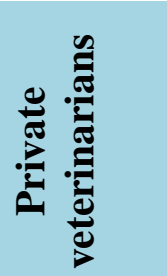 & & 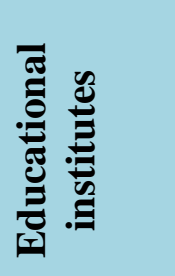 & שֶ, & 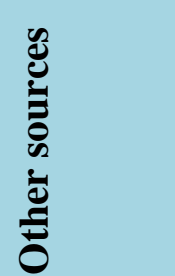 \\
\hline & & $n=45$ & $\mathrm{n}=\mathbf{2 2}$ & $\mathrm{n}=120$ & $\mathrm{n}=120$ & $\mathrm{n}=120$ & $\mathrm{n}=120$ & $\mathrm{n}=120$ & $\mathrm{n}=120$ \\
\hline 1 & Curative services & $\begin{array}{c}34 \\
(75.55)\end{array}$ & $\begin{array}{c}18 \\
(81.82)\end{array}$ & $\begin{array}{c}56 \\
(46.67)\end{array}$ & $\begin{array}{c}119 \\
(99.17)\end{array}$ & $\begin{array}{c}56 \\
(46.67)\end{array}$ & $\begin{array}{c}1 \\
(0.83)\end{array}$ & $\begin{array}{c}0 \\
(0.00)\end{array}$ & $\begin{array}{c}0 \\
(0.00)\end{array}$ \\
\hline 2 & Preventive services & $\begin{array}{c}38 \\
(84.44)\end{array}$ & $\begin{array}{c}18 \\
(81.82)\end{array}$ & $\begin{array}{c}80 \\
(66.67)\end{array}$ & $\begin{array}{c}114 \\
(95.00)\end{array}$ & $\begin{array}{c}51 \\
(42.50)\end{array}$ & $\begin{array}{c}2 \\
(1.67)\end{array}$ & $\begin{array}{c}8 \\
(6.67)\end{array}$ & $\begin{array}{c}0 \\
(0.00)\end{array}$ \\
\hline 3 & Medicines & $\begin{array}{c}36 \\
(80.00)\end{array}$ & $\begin{array}{c}18 \\
(81.82)\end{array}$ & $\begin{array}{c}79 \\
(65.83)\end{array}$ & $\begin{array}{c}117 \\
(97.50)\end{array}$ & $\begin{array}{c}51 \\
(42.50)\end{array}$ & $\begin{array}{c}1 \\
(0.83)\end{array}$ & $\begin{array}{c}118 \\
(98.33)\end{array}$ & $\begin{array}{c}0 \\
(0.00)\end{array}$ \\
\hline 4 & Fodder seeds and slips & $\begin{array}{c}15 \\
(33.33)\end{array}$ & $\begin{array}{c}2 \\
(9.09)\end{array}$ & $\begin{array}{c}2 \\
(1.67)\end{array}$ & $\begin{array}{c}0 \\
(0.00)\end{array}$ & $\begin{array}{c}0 \\
(0.00)\end{array}$ & $\begin{array}{c}11 \\
(9.17)\end{array}$ & $\begin{array}{c}0 \\
(0.00)\end{array}$ & $\begin{array}{c}71 \\
(59.17)\end{array}$ \\
\hline 5 & Concentrate cattle feed & $\begin{array}{c}33 \\
(73.33)\end{array}$ & $\begin{array}{c}10 \\
(45.45)\end{array}$ & $\begin{array}{c}0 \\
(0.00)\end{array}$ & $\begin{array}{c}0 \\
(0.00)\end{array}$ & $\begin{array}{c}0 \\
(0.00)\end{array}$ & $\begin{array}{c}0 \\
(0.00)\end{array}$ & $\begin{array}{c}0 \\
(0.00)\end{array}$ & $\begin{array}{c}76 \\
(63.33)\end{array}$ \\
\hline 6 & $\begin{array}{l}\text { Mineral mixture and } \\
\text { other supplements }\end{array}$ & $\begin{array}{c}33 \\
(73.33)\end{array}$ & $\begin{array}{c}2 \\
(9.09)\end{array}$ & $\begin{array}{c}19 \\
(15.83)\end{array}$ & $\begin{array}{c}10 \\
(8.33)\end{array}$ & $\begin{array}{c}1 \\
(0.83)\end{array}$ & $\begin{array}{c}5 \\
(4.17)\end{array}$ & $\begin{array}{c}69 \\
(57.50)\end{array}$ & $\begin{array}{c}0 \\
(0.00)\end{array}$ \\
\hline 7 & Credit facilities & $\begin{array}{c}5 \\
(11.11)\end{array}$ & $\begin{array}{c}1 \\
(4.54)\end{array}$ & $\begin{array}{c}0 \\
(0.00)\end{array}$ & $\begin{array}{c}0 \\
(0.00)\end{array}$ & $\begin{array}{c}0 \\
(0.00)\end{array}$ & $\begin{array}{c}0 \\
(0.00)\end{array}$ & $\begin{array}{c}0 \\
(0.00)\end{array}$ & $\begin{array}{c}15 \\
(12.50)\end{array}$ \\
\hline 8 & Insurance facilities & $\begin{array}{c}26 \\
(57.78)\end{array}$ & $\begin{array}{c}1 \\
(4.54)\end{array}$ & $\begin{array}{c}26 \\
(21.67)\end{array}$ & $\begin{array}{c}0 \\
(0.00)\end{array}$ & $\begin{array}{c}0 \\
(0.00)\end{array}$ & $\begin{array}{c}0 \\
(0.00)\end{array}$ & $\begin{array}{c}0 \\
(0.00)\end{array}$ & $\begin{array}{c}0 \\
(0.00)\end{array}$ \\
\hline 9 & AI services & $\begin{array}{c}26 \\
(57.78)\end{array}$ & $\begin{array}{c}15 \\
(68.18)\end{array}$ & $\begin{array}{c}41 \\
(34.17)\end{array}$ & $\begin{array}{c}69 \\
(57.50)\end{array}$ & $\begin{array}{c}91 \\
(75.83)\end{array}$ & $\begin{array}{c}0 \\
(0.00)\end{array}$ & $\begin{array}{c}0 \\
(0.00)\end{array}$ & $\begin{array}{c}0 \\
(0.00)\end{array}$ \\
\hline 10 & $\begin{array}{l}\text { Training and advisory } \\
\text { services }\end{array}$ & $\begin{array}{c}27 \\
(60.00)\end{array}$ & $\begin{array}{c}4 \\
(18.18)\end{array}$ & $\begin{array}{c}34 \\
(28.33)\end{array}$ & $\begin{array}{c}0 \\
(0.00)\end{array}$ & $\begin{array}{c}0 \\
(0.00)\end{array}$ & $\begin{array}{c}7 \\
(5.83)\end{array}$ & $\begin{array}{c}0 \\
(0.00)\end{array}$ & $\begin{array}{c}0 \\
(0.00)\end{array}$ \\
\hline
\end{tabular}

Figures in parenthesis indicate percentage 
Table.2 Perceived effectiveness of health services of dairy service delivery systems

\begin{tabular}{|l|c|c|c|c|}
\hline \multicolumn{1}{|c|}{ Service provider } & Regularity & Timeliness & Quality & $\begin{array}{c}\text { Cost } \\
\text { effectiveness }\end{array}$ \\
\hline Dairy co-operative & \multicolumn{3}{|c|}{ Curative services } \\
\hline Private integrators & 155.88 & 188.24 & 273.53 & 288.24 \\
\hline Public department & 292.86 & $\mathbf{2 9 6 . 4 3}$ & 289.29 & $\mathbf{3 0 0 . 0 0}$ \\
\hline Private veterinarians & $\mathbf{2 9 9 . 1 6}$ & 247.06 & $\mathbf{2 9 9 . 1 6}$ & 142.02 \\
\hline Para-veterinarians & 265.45 & 252.73 & 200.00 & 158.18 \\
\hline & \multicolumn{2}{|c|}{ Preventive services } \\
\hline Dairy co-operative & 160.53 & 189.47 & 276.32 & 292.11 \\
\hline Private integrators & 166.67 & 177.78 & 283.33 & 222.22 \\
\hline Public department & 271.25 & $\mathbf{2 7 3 . 7 5}$ & 285.00 & $\mathbf{3 0 0 . 0 0}$ \\
\hline Private veterinarians & $\mathbf{2 9 9 . 1 2}$ & 244.74 & $\mathbf{2 9 9 . 1 2}$ & 141.23 \\
\hline Para-veterinarians & 264.71 & 252.94 & 200.00 & 160.78 \\
\hline & & Medicines & & \\
\hline Dairy co-operative & 158.33 & 188.89 & 275.00 & 291.67 \\
\hline Private integrators & 166.67 & 177.78 & 283.33 & 222.22 \\
\hline Public department & 273.42 & 275.95 & 284.81 & $\mathbf{3 0 0 . 0 0}$ \\
\hline Private veterinarians & $\mathbf{2 9 9 . 1 5}$ & 246.15 & $\mathbf{2 9 9 . 1 5}$ & 141.88 \\
\hline Para-veterinarians & 266.67 & 254.90 & 201.96 & 158.82 \\
\hline Pharmacies & 297.46 & $\mathbf{2 9 8 . 3 1}$ & 296.61 & 141.53 \\
\hline
\end{tabular}

Table.3 Perceived effectiveness of supply of fodder seeds and slips by different dairy service delivery systems

\begin{tabular}{|l|c|c|c|c|c|c|}
\hline \multicolumn{1}{|c|}{ Service provider } & Regularity & Timeliness & Quality & Quantity & Variety & $\begin{array}{c}\text { Cost } \\
\text { effectiveness }\end{array}$ \\
\hline Dairy co-operative & $\mathbf{2 2 6 . 6 7}$ & $\mathbf{2 2 0 . 0 0}$ & 286.67 & 280.00 & 280.00 & 200.00 \\
\hline $\begin{array}{l}\text { Educational } \\
\text { institutes }\end{array}$ & 209.09 & 190.91 & $\mathbf{2 9 0 . 9 1}$ & 263.64 & $\mathbf{2 9 0 . 9 1}$ & 209.09 \\
\hline Neighbours & 197.18 & 191.55 & 259.15 & $\mathbf{2 8 8 . 7 3}$ & 256.34 & $\mathbf{3 0 0 . 0 0}$ \\
\hline
\end{tabular}

Table.4 Perceived effectiveness of supply of concentrate feed by different dairy service delivery systems

\begin{tabular}{|l|c|c|c|c|c|c|}
\hline Service provider & Regularity & Timeliness & Quality & Quantity & $\begin{array}{c}\text { Type of } \\
\text { feed }\end{array}$ & $\begin{array}{c}\text { Cost } \\
\text { effectiveness }\end{array}$ \\
\hline Dairy co-operative & 200.00 & 209.09 & 281.82 & 248.48 & 281.82 & 187.88 \\
\hline Private integrators & 270.00 & 280.00 & 270.00 & 290.00 & 270.00 & $\mathbf{2 2 2 . 2 2}$ \\
\hline $\begin{array}{l}\text { Commercial feed } \\
\text { agencies }\end{array}$ & $\mathbf{2 9 7 . 3 7}$ & $\mathbf{2 9 7 . 3 7}$ & $\mathbf{2 9 8 . 6 8}$ & $\mathbf{2 9 8 . 6 8}$ & $\mathbf{2 9 8 . 6 8}$ & 123.68 \\
\hline
\end{tabular}


Table.5 Perceived effectiveness of supply of mineral mixture by different dairy service delivery systems

\begin{tabular}{|l|c|c|c|c|c|}
\hline \multicolumn{1}{|c|}{ Service provider } & Regularity & Timeliness & Quality & Quantity & $\begin{array}{c}\text { Cost } \\
\text { effectiveness }\end{array}$ \\
\hline Dairy co-operative & 130.30 & 124.24 & 236.36 & 215.15 & 248.48 \\
\hline Public department & 247.37 & 257.89 & 273.68 & 263.16 & $\mathbf{2 9 4 . 7 4}$ \\
\hline Private veterinarians & 120.00 & 130.00 & 290.00 & 180.00 & 210.00 \\
\hline Pharmacies & $\mathbf{3 0 0 . 0 0}$ & $\mathbf{2 9 8 . 5 3}$ & $\mathbf{3 0 0 . 0 0}$ & $\mathbf{3 0 0 . 0 0}$ & 116.18 \\
\hline
\end{tabular}

Table.6 Perceived effectiveness of credit facilities rendered by different dairy service delivery systems

\begin{tabular}{|l|c|c|c|c|c|}
\hline Service provider & Accessibility & Timeliness & $\begin{array}{c}\text { Interest } \\
\text { rate }\end{array}$ & $\begin{array}{c}\text { Administrative } \\
\text { procedure }\end{array}$ & $\begin{array}{c}\text { Flexibility in } \\
\text { repayment }\end{array}$ \\
\hline Dairy co-operative & $\mathbf{3 0 0 . 0 0}$ & $\mathbf{3 0 0 . 0 0}$ & $\mathbf{3 0 0 . 0 0}$ & $\mathbf{3 0 0 . 0 0}$ & $\mathbf{3 0 0 . 0 0}$ \\
\hline Banks & 286.67 & 220.00 & 246.67 & 233.33 & 273.33 \\
\hline
\end{tabular}

Table.7 Perceived effectiveness of insurance facilities offered by different dairy service delivery systems

\begin{tabular}{|l|c|c|c|c|c|}
\hline Service provider & Accessibility & Coverage & $\begin{array}{c}\text { Premium } \\
\text { charges }\end{array}$ & $\begin{array}{c}\text { Administrative } \\
\text { procedure }\end{array}$ & Claiming \\
\hline Dairy co-operative & $\mathbf{3 0 0 . 0 0}$ & $\mathbf{3 0 0 . 0 0}$ & $\mathbf{3 0 0 . 0 0}$ & $\mathbf{3 0 0 . 0 0}$ & 288.89 \\
\hline Public department & $\mathbf{3 0 0 . 0 0}$ & 296.15 & $\mathbf{3 0 0 . 0 0}$ & $\mathbf{3 0 0 . 0 0}$ & $\mathbf{3 0 0 . 0 0}$ \\
\hline
\end{tabular}

Table.8 Perceived effectiveness of artificial insemination services by different dairy service delivery systems

\begin{tabular}{|l|c|c|c|c|c|}
\hline Service provider & Timeliness & $\begin{array}{c}\text { Semen of } \\
\text { varied breeds }\end{array}$ & $\begin{array}{c}\text { Quality } \\
\text { of service }\end{array}$ & $\begin{array}{c}\text { Success } \\
\text { rate }\end{array}$ & $\begin{array}{c}\text { Cost } \\
\text { effectiveness }\end{array}$ \\
\hline Dairy co-operative & 253.85 & 250.00 & 273.08 & 284.62 & 284.62 \\
\hline Private integrators & 246.67 & 260.00 & 293.33 & 280.00 & 260.00 \\
\hline Public department & $\mathbf{2 9 7 . 5 6}$ & $\mathbf{2 8 2 . 9 3}$ & $\mathbf{3 0 0 . 0 0}$ & $\mathbf{2 9 2 . 6 8}$ & $\mathbf{3 0 0 . 0 0}$ \\
\hline Private veterinarians & 284.06 & 257.97 & 297.10 & 286.96 & 136.23 \\
\hline Para-veterinarians & 282.42 & 257.14 & 275.82 & 264.84 & 197.80 \\
\hline
\end{tabular}

Table.9 Perceived effectiveness of training and advisory services by different dairy service delivery systems

\begin{tabular}{|l|c|c|c|c|c|}
\hline Service provider & $\begin{array}{c}\text { Knowledge } \\
\text { and skills }\end{array}$ & Infrastructure & $\begin{array}{c}\text { Staff } \\
\text { attitude }\end{array}$ & Flexibility & Need basis \\
\hline Dairy co-operative & $\mathbf{2 9 2 . 5 9}$ & $\mathbf{2 6 6 . 6 7}$ & $\mathbf{2 9 6 . 3 0}$ & $\mathbf{2 8 1 . 4 8}$ & $\mathbf{2 8 8 . 8 9}$ \\
\hline Public department & 247.06 & 250.00 & 261.76 & 252.94 & 244.12 \\
\hline
\end{tabular}


Table.10 Overall perceived effectiveness of different dairy service delivery systems

\begin{tabular}{|c|c|c|c|c|c|c|c|c|c|}
\hline $\begin{array}{l}\text { S. } \\
\text { No. }\end{array}$ & Type of services & d & 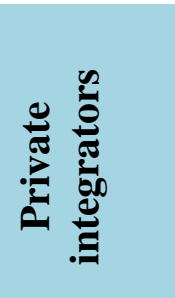 & 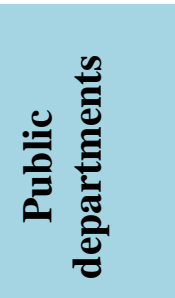 & 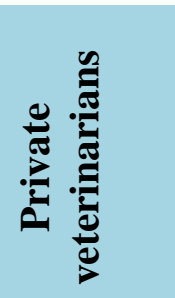 & • & 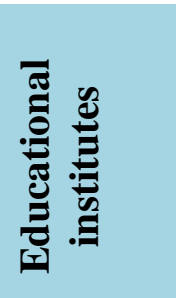 & & 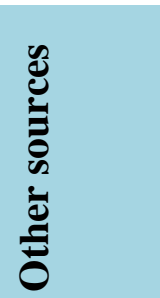 \\
\hline 1 & Curative services & $\begin{array}{l}226.47 \\
\text { (III) }\end{array}$ & $\begin{array}{c}212.50 \\
(\mathrm{~V})\end{array}$ & $\begin{array}{l}294.15 \\
\text { (I) }\end{array}$ & $\begin{array}{l}246.85 \\
\text { (II) }\end{array}$ & $\begin{array}{c}219.09 \\
(I V)\end{array}$ & -- & -- & -- \\
\hline 2 & Preventive services & $\begin{array}{l}229.61 \\
\text { (III) }\end{array}$ & $\begin{array}{l}212.50 \\
(\mathrm{~V})\end{array}$ & $\begin{array}{l}282.28 \\
\text { (I) }\end{array}$ & $\begin{array}{l}246.05 \\
\text { (II) }\end{array}$ & $\begin{array}{c}4 \\
(219.61)\end{array}$ & -- & -- & -- \\
\hline 3 & Medicines & $\begin{array}{l}228.47 \\
\text { (IV) }\end{array}$ & $\begin{array}{l}212.50 \\
(\mathrm{VI})\end{array}$ & $\begin{array}{l}283.55 \\
\text { (I) }\end{array}$ & $\begin{array}{l}246.58 \\
\text { (III) }\end{array}$ & $\begin{array}{c}220.59 \\
(\mathrm{~V})\end{array}$ & -- & $\begin{array}{l}262.80 \\
\text { (II) }\end{array}$ & -- \\
\hline 4 & Fodder seeds and slips & $\begin{array}{c}248.89 \\
\text { (I) }\end{array}$ & -- & -- & -- & -- & $\begin{array}{c}242.38 \\
\text { (III) }\end{array}$ & -- & $\begin{array}{l}248.83 \\
\text { (II) }\end{array}$ \\
\hline 5 & Concentrate cattle feed & $\begin{array}{l}234.85 \\
\text { (III) }\end{array}$ & $\begin{array}{l}267.04 \\
\text { (II) }\end{array}$ & -- & -- & -- & -- & -- & $\begin{array}{l}269.08 \\
\text { (I) }\end{array}$ \\
\hline 6 & $\begin{array}{l}\text { Mineral mixture and } \\
\text { other supplements }\end{array}$ & $\begin{array}{l}190.91 \\
\text { (III) }\end{array}$ & -- & $\begin{array}{l}267.37 \\
\text { (I) }\end{array}$ & $\begin{array}{c}186.00 \\
(\mathrm{IV})\end{array}$ & -- & -- & $\begin{array}{l}262.94 \\
\text { (II) }\end{array}$ & -- \\
\hline 7 & Credit facilities & $\begin{array}{l}300.00 \\
\text { (I) }\end{array}$ & -- & -- & -- & -- & -- & -- & $\begin{array}{l}252.00 \\
\text { (II) }\end{array}$ \\
\hline 8 & Insurance facilities & $\begin{array}{l}297.78 \\
\text { (II) }\end{array}$ & -- & $\begin{array}{l}299.23 \\
\text { (I) }\end{array}$ & -- & -- & -- & -- & -- \\
\hline 9 & AI services & $\begin{array}{l}269.23 \\
\text { (II) }\end{array}$ & $\begin{array}{l}268.00 \\
\text { (III) }\end{array}$ & $\begin{array}{l}294.63 \\
\text { (I) }\end{array}$ & $\begin{array}{c}252.46 \\
(\mathrm{~V})\end{array}$ & $\begin{array}{l}255.60 \\
\text { (IV) }\end{array}$ & -- & -- & -- \\
\hline 10 & $\begin{array}{l}\text { Training and advisory } \\
\text { services }\end{array}$ & $\begin{array}{c}285.19 \\
\text { (I) }\end{array}$ & -- & $\begin{array}{l}252.18 \\
\text { (II) }\end{array}$ & -- & -- & -- & -- & -- \\
\hline
\end{tabular}




\section{Mineral mixture}

It can be inferred from the table 5 that pharmacies were perceived as highly effective in terms of their regularity (300.00), timeliness (298.53), quality (300.00) and quantity (300.00). However, the public department were perceived to be cost effective (294.74) as they were distributing the TANUVAS mineral mixture at free of cost for trial as part of schemes functioning during the study period.

\section{Credit facilities}

All the dairy farmers perceived that the credit facilities rendered by dairy co-operative were effective than commercial and cooperative banks (Table 6). The credit facilities of dairy co-operative were accessible to all the members in time at nominal interest rate with minimum administrative procedures and flexible repayment schedule.

\section{Insurance}

The dairy farmers perceived that dairy cooperative and public departments were equally effective in terms of their accessibility, premium charges and administrative procedure for insurance. However, they perceived the claiming process was difficult in dairy co-operative (Table 7).

\section{Artificial insemination services}

From table 8, it can be inferred that artificial insemination service provided by the public department was perceived as overwhelmingly effective for all the indicators measured viz. timeliness (297.56), semen of varied breeds (282.93), quality of service (300.00), success rate (292.68) and cost effectiveness (300.00). The state animal husbandry department has organised and systematic time schedule of activities with subsidized rate which resulted in delivering effective artificial insemination service to the dairy farmers.

\section{Training and advisory services}

Based on the weighted percentage scores presented in the table 9, it could be inferred that the dairy farmers perceived the training and advisory services provided by dairy cooperative was most effective in terms of knowledge and skills (292.59), infrastructure (266.67), staff attitude (296.30), flexibility (281.48) and need basis (288.89).

While the public department deals with all livestock and poultry, the dairy co-operative concentrate only on dairying resulted in effective service delivery. Rathod et al., (2011) stated that significant number of competent and reliable human resource team delivered extension services effectively in the form of training, advisory service, farmers educational tour, farm visits, exhibitions etc. The respondents perceived that the relevant and need based extension services were available from dairy co-operative was good.

\section{Overall perceived effectiveness of the dairy service delivery systems}

The overall perceived effectiveness of dairy service delivery systems were studied based on their weighted percentage mean score. From the table 10, it is evident that public department was ranked first in providing curative services (294.65), preventive services (282.38), medicines (283.55), mineral mixture (267.37), insurance (299.23) and artificial insemination services (294.63). It is due to the fact that public department is operating throughout the year and most of the services are rendered at free of cost which makes it more effective. Insurance for the dairy animals was partially subsidized by the government agencies might be the reason for perceiving them as most effective. 
The service of dairy co-operative was perceived as effective in supplying fodder seeds and slips (248.89), rendering credit facilities to their members (300.00) and providing training and advisory services (285.19). Dairy co-operative maintained stock of good quality fodder seeds and slips of different improved varieties and it was distributed to their members regularly at nominal cost. The members of the dairy cooperative were also given periodical training in improved dairy farming and fodder cultivation and they were also taken for field visits regularly. The credit facilities were arranged by the co-operative at a nominal rate of interest and it was repaid from the payment of milk.

With the weighted percentage mean score of 269.08, the commercial feed agencies were perceived as the effective service provider in supplying commercial concentrate feed to the dairy farmers which was not adequately available from other systems. Though the service was not cost effective, their regularity, quality and timeliness in supply made it as the best system in the market.

Most of the respondents predominantly availed the health services from the private veterinarians than other dairy service delivery systems. The para-veterinarians are the major source for the respondents for availing artificial insemination services. The respondents were availing the services such as fodder seeds, fodder slips, concentrate cattle feed, mineral mixture, insurance and training and advisory services from dairy cooperative.

The public department was ranked first in providing curative services, preventive services, medicines, mineral mixture, insurance and artificial insemination services effectively. While the dairy co-operative was perceived to be effective in supplying fodder seeds and slips; credit facilities; and training and advisory services. The commercial feed agencies were perceived as effective service provider in supplying commercial concentrate feed than others.

The private integrators and dairy cooperatives need to take necessary efforts to provide timely and cost effective services to increase the net profit by cutting down the health and breeding expenses of the dairy farmers. Hence, there is a need to restructure the delivery mechanism of private integrators and dairy co-operatives for efficient and essential service delivery in tandem with the requirement of rural dairy farmers.

\section{References}

Annual Report 2016-17 (2017) Department of Animal Husbandry, Dairying and Fisheries, Ministry of Agriculture and Farmers Welfare, Government of India.

Bardhan, D., Kumar, S. and Singh, R.K. (2015). Delivery of Animal Healthcare Services in Uttar Pradesh: Present Status, Challenges and Opportunities, Agricultural Economics Research Review. 28 (Conference Number):127136.

Kathiravan, G., Thirunavukkarasu, D. and Selvam, S. (2011). Time, costs and farmers' perceptions: The case of livestock service delivery in Tamilnadu. Veterinary World, 4 (5): 209-212.

Pallavi, P.M., Kumar, S. and Singh, Y.P. (2011). Preference of service providers for the veterinary service-a case study of Sangli District of Maharastra state, India. Veterinary World, 2011, 4 (3):106-108.

Policy Note 2017-18 (2017) Department of Animal Husbandry, Dairying \& Fisheries, Government of Tamil Nadu.

Rathod, P., Nikam, T. R., Landge, S. and Hatey, A. (2012). Farmers Perception 
towards Livestock Extension Service: A Case Study. Indian Research Journal of Extension Education, 2 (Special Issue): 1-5.

Rathod, P., Nikam, T. R., Landge, S. and Hatey, A., (2011). SWOT Analysis of Dairy Cooperatives: A Case Study of Western Maharashtra. International Journal of Research in Commerce and Management. 2 (8): 35-41.
Sharma, K. (2001). Functioning of milk cooperatives in Ludhiana district of Punjab. Journal of Extension Education, 37 (1\&2): 69-73.

Umali, D., Feder, G. and De Haan, C. (1992). The balance between public and private sector activities in the delivery of livestock services. World Bank Discussion Papers \#1 No.63, Washington, D.C., U.S.A.

\section{How to cite this article:}

Karthikeyan, S., M.C. Arunmozhi Devi, N. Narmatha and Uma, V. 2018. Perceived Effectiveness of Dairy Service Delivery Systems in Namakkal District of Tamil Nadu, India. Int.J.Curr.Microbiol.App.Sci. 7(05): 337-347. doi: https://doi.org/10.20546/ijcmas.2018.705.043 